\title{
Guest editorial: special issue on social media and interactive technologies
}

\author{
Timothy K. Shih ${ }^{1} \cdot$ Lin Hui $^{2}$ - Somchoke Ruengittinun ${ }^{3} \cdot$ Qing $\mathrm{Li}^{4}$ \\ Published online: 26 March 2019 \\ (C) Springer Science+Business Media, LLC, part of Springer Nature 2019
}

Events recently in cyber-world can be modeled as a spatiotemporal continuity which is populated by computing devices, communication channels, and multimodal interactions. Contemporary ubiquitous devices unleash the boundary of one-to-one human-computer interaction becoming a de facto style of facilitating social events that participants use several distributed devices through multimodal interactions. The aim of this special issue is to solicit technical contributions regarding theoretical and practical aspects of scenario concept, architecture design, prototype development, implementation, and demonstration on state-of-the-art technologies and new research findings in multimedia, such as location/context adaptation, inter-device interaction/reaction, social media analysis, and media/data communication, etc.

We organized this special issue in conjunction with the call for papers and participations of the 10th International Conference on Ubi-media Computing and Workshops: Networks, Systems, and Applications, Pattaya, Thailand, August 3, 2017. Among 56 papers submitted to Ubi-media'17, there were only two accepted papers were recommended to this special issue.

Lin Hui

121678@mail.tku.edu.tw

Timothy K. Shih

timothykshih@gmail.com

Somchoke Ruengittinun

somchoke.r@gmail.com

Qing Li

itqli@ cityu.edu.hk

1 Department of Computer Science and Information Engineering, National Central University, No. 300, Zhong-da Road, Zhong-li District, Taoyuan 32001, Taiwan

2 Department of Innovative Information and Technology, Tamkang University, No.180, Linwei Rd., Jiaoxi Township, Yilan County 26247, Taiwan

3 Department of Computer Science, Kasetsart University, 50 Ngam Wong Wan Rd, Lat Yao Chatuchak, Bangkok 10900, Thailand

4 Department of Computer Science, City University of Hong Kong, Tat Chee Avenue, Kowloon, Hong Kong, SAR, China 
The special issue received 53 submissions, all of which were asked to contain the significant extensions from their previously conference versions, and to be reviewed again by three reviewers. Eventually, we accepted 12 papers. Each of these papers was finalized with two more substantial revisions and then for publication according to the reviewers' comments, suggestions, and the Editor-in-Chief's decision.

These 12 accepted articles now provide novel, interesting, and useful results, covering recommendation $[1,2]$, learning $[3,4]$, augmented reality (AR) $[5,6]$, social network analysis $[7,8,9]$, artificial intelligence (AI) [10, 11] and related media application [12].

The accepted articles in this special issue are as follows:

[1] Zhiyuan Zhang, Yun Liu, Zhenjiang Zhang, Bo Shen

Fused matrix factorization with multi-tag, social and geographical influences for POI recommendation

[2] Hung-Yi Gau, Yi-Shu Lu, Wen-Yueh Shih, Kuan-Chieh Chung, Jiun-Long Huang

On successive point-of-interest recommendation

[3] Lihong Han, Gaofeng Zhang, Binbin Yong, Qiang He, Fang Feng, Qingguo Zhou

Statistical study of characteristics of online reading behavior networks in university digital library

[4] Ming Zhang, Jile Zhu, Zhuo Wang, Yunfan Chen

Providing personalized learning guidance in MOOCs by multi-source data analysis

[5] Shih-Wei Sun, Yi-Shan Lan

Augmented reality displaying scheme in a smart glass based on relative object positions and orientation sensors

[6] Ching-Sheng Wang

An AR mobile navigation system integrating indoor positioning and content recommendation services

[7] Kiichi Tago, Kosuke Takagi, Seiji Kasuya, Qun Jin

Analyzing influence of emotional tweets on user relationships using Naive Bayes and dependency parsing

[8] Yi-Cheng Chen

A novel algorithm for mining opinion leaders in social networks

[9] Wei-Yun Hsu, Hui-Huang Hsu, Vincent S. Tseng

Springer 
Discovering negative comments by sentiment analysis on Web forum

[10] Ying Liu, Yanan Peng, Kengpang Lim, Nam Ling

A novel image retrieval algorithm based on transfer learning and fusion features

[11] Haiman Tian, Yudong Tao, Samira Pouyanfar, Shu-Ching Chen, Mei-Ling Shyu

Multimodal deep representation learning for video classification

[12] Qianhui Men, Howard Leung, Yang Yang

Self-feeding frequency estimation and eating action recognition from skeletal representation using Kinect

This editorial message not only delineates the paper submission and reviewing process, but also sincerely appreciates the authors' patience for paper revisions, the reviewers' devotion and time commitment which is necessary to assure the high quality articles. Finally, we would like to thank Dr. Yanchun Zhang, the Editor-in-Chief, and the journal staffs of WWWJ for providing all of the important advices and suggestions during entire process in the completion of this special issue.

Publisher's note Springer Nature remains neutral with regard to jurisdictional claims in published maps and institutional affiliations. 